EASD

Procedia
EURODYN 2020

XI International Conference on Structural Dynamics M. Papadrakakis, M. Fragiadakis, C. Papadimitriou (eds.) Athens, Greece, 23-26 November 2020

\title{
A NEW SEMI-ANALYTICAL APPROACH FOR DYNAMIC PILE-SOIL INTERACTION PROBLEM
}

\author{
Kenny F. Conto ${ }^{1}$, Robert Arcos ${ }^{2}$, Cecília Parente ${ }^{3}$, Pedro Alves Costa ${ }^{4}$ and Jordi Romeu ${ }^{1}$ \\ ${ }^{1}$ Acoustical and Mechanical Engineering Laboratory (LEAM), Universitat Politècnica de Catalunya \\ (UPC) \\ Carrer Colom, 11, 08222 Terrassa (Barcelona), Spain \\ e-mail: kenny.fernando.conto,jordi.romeu.upc@upc.edu \\ ${ }^{2}$ Serra Húnter Fellow, Acoustical and Mechanical Engineering Laboratory (LEAM), Universitat \\ Politècnica de Catalunya (UPC) \\ Carrer Colom, 11, 08222 Terrassa (Barcelona), Spain \\ e-mail: robert.arcos@upc.edu \\ ${ }^{3}$ Mota-Engil Engenharia e Construção, S.A. \\ Rua do Rego Lameiro, 38, 4300-454 Porto, Portugal \\ e-mail: cparente@fe.up.pt \\ ${ }^{4}$ CONSTRUCT, Faculty of Engineering (FEUP), University of Porto \\ Rua Dr. Roberto Frias, s/n 4200-465 Porto, Portugal \\ e-mail: pacosta@fe.up.pt
}

Keywords: Dynamic pile-soil interaction, Pile foundations, substructuring method.

\begin{abstract}
This paper proposes a new semi-analytical approach for the computation of the dynamic response of a pile embedded in a half-space when it is subjected to a vertical harmonic load applied at the pile head or to loading from an incident wave field. The proposed approach considers the soil as an elastic half-space medium. In order to capture all essential dynamic behaviour of the pile-soil interaction avoiding large computational efforts, the system is modelled using the substructuring method in which the displacements and forces in a set of uniformly distributed points along the pile shaft are compatibilized with another set of coupling points in the soil also uniformly distributed along the virtual interaction area between the pile and the soil. The paper aims to study the accuracy and computational efficiency of this approach in comparison with two other methodologies: a numerical approach based on axisymmetric finite elements and perfect-matched layers, and against the approximate analytical approach based on linear elasticity developed by Novak. These comparisons reveal that the dynamic response for driving-point, far-field or incident wave-field problems can be predicted with acceptable accuracy and computational efficiency when the number of interaction points in the system increases.
\end{abstract}

ISSN:2311-9020 (C) 2020 The Authors. Published by EASD Procedia.

Peer-review under responsibility of the Organizing Committee of EURODYN 2020.

doi: $10.47964 / 1120.9230 .19042$ 


\section{INTRODUCTION}

Ground-borne vibration is an important cause of annoyance to nearby buildings inhabitants, malfunction on precision devices in surrounding facilities or even structural damage. These negative effects occurs when the ground-borne vibration incident wave-field excites the building structure through its foundations, being piled foundations one of the most common types. There are different models used to study the dynamic response of a single pile embedded in a half-space, which can be classified into three broad groups [6]. First group is based on the dynamic Winkler foundation approach, attributed as earliest building foundations dynamic models. In that method, the soil medium is modelled by infinitesimally, thin, independent, horizontal layers, meaning that waves can be only propagated in horizontal direction. This approach is adopted in the Novak's benchmark research [10], where the dynamic response of single piles is found throughout an Euler-Bernoulli beam and axial rod models of the pile for the lateral and vertical responses, respectively, which include the soil reaction directly in their differential equations of motion. The second type of methods are based on modelling the soil as an elastic medium. They are proposed to account for wave propagation in the soil in all directions. A relevant model of this method was presented by Kaynia and Kausel [3], where a general formulation for the prediction of the dynamic response of single piles and pile groups embedded in a layered half-space is presented. In this method the soil is also modelled as an elastic half-space while the piles are modelled as Euler-Bernoulli beams and axial rods. Furthermore, forces and displacements of each substructure (soil and pile) are compatibilised in a set of coupling points in order to compute the response of the system. These coupling points are defined when the whole traction acting on the virtual soil-pile interface is segmented along the pile shaft. Where, each segment is identified by a node and placed in its center. Therefore, each node represents either the load acting on the lateral surface of the pile (called barrel load distribution), or the load which is related to the pile-tip surface (called disk load distribution). Recently, the PiP model developed by Forrest [2] was adapted by Kuo et al. [7, 8] in order to create a three-dimensional single-pile dynamic model, which is formulated in the wavenumber domain and uses mirrorimage sources to simulate a half-space and the finite length of the piles. Ntotsios et al. [11] developed two methods for the dynamic response of piled foundations where the approach for the coupling technique is similar to the one proposed by Kaynia in [3]. Euler beam and axial rod formulations are again used to model the pile. The dynamic compatibility between the pile and the soil is performed by distributing the displacements and forces in discrete points along the pile shaft over an annulus on the soil, with mean radius equal to the pile radius and a thickness of one-tenth of that, except for the pile-tip, where the distribution is done over a circular disk with the same radius than the pile. On their first method, the response of the soil is computed using the thin layer method (TLM), while in the second approach, the boundary element method (BEM) is used to model the soil medium with the pile cavity. Finally, numerical approaches are also an interesting modelling option for piled foundations. With the finite element method (FEM) $[5,4]$, the non-linear behaviour of the soil and its interaction with the pile can be introduced. However, the computation of FEM models requires large computational efforts, mostly when high frequency analysis is performed. Furthermore, the FEM applied to wave propagation problems in the soil requires the implementation of energy-absorbing boundaries to simulate an unbounded medium [1]. The BEM is another alternative to be used for this type of problems in order to model the soil, where the use of energy-absorbing boundaries is not required.

In some piled foundation problems where only the pile-driving response is required, the Winkler foundation approach could be enough to compute the dynamic response. When the soil 
response is required or when the coupled response between piles is desired to be computed, the low accuracy of this method for these problems releases the numerical approach as the most suitable method to compute the dynamic response without decreasing accuracy. However, the usage of these methods is usually complex and they inherently have high computational requirements. For these reasons, the aim of this paper is presenting a new semi-analytical approach for the computation of the dynamic response of a single pile embedded in a half-space when it is subjected to a vertical harmonic load applied at the pile head or to the action of an incident wave-field. The benefits are its computational efficiency keeping high accuracy levels and the simplicity of its implementation in a computer algorithm, becoming a suitable tool for design use by practising engineers.

\section{SINGLE PILE-SOIL MODEL}

The current section starts describing the assumptions adopted in the present work. Then, the resulting formulation of the pile-soil model presented here is described. This pile-soil model is mainly constructed using dynamic substructuring techniques. Thus, the dynamic behaviour of each substructure (the pile and the soil) is analysed separately to be later assembled using a coupling procedure. The assumptions followed here are:

- The pile is assumed to be perfectly elastic and it is modelled as a structural column member for the axial response. Lateral motion is not considered in the present work.

- The soil is considered to be a perfectly elastic homogeneous half-space.

- The non-linear effects associated to the soil-pile contact interaction, high strain levels or liquefaction of the soil are neglected.

- The coupling of sub-systems is performed in the basis of equilibrium of forces and compatibility of displacement conditions.

Fig. 1 illustrates the system of a single piled foundation embedded in a homogeneous halfspace, which is the problem considered in this study. The system is subjected to a external time-harmonic vertical point load applied either at the pile head or on the soil. In the framework

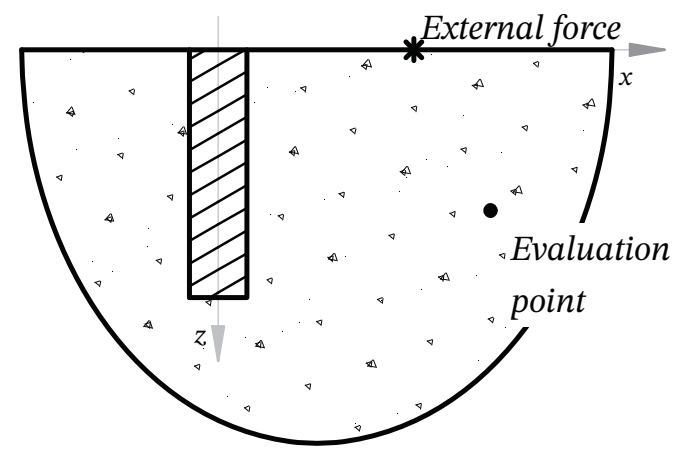

Figure 1: Single pile model.

of dynamic substructuring, the coupling between the pile and the soil is proposed to be done in a set of uniformly distributed coupling points. To do so, the soil-pile interaction traction, 
distributed along the the soil-pile interface, is divided into $N+1$ segments (see Fig. 2a) and each of these segments is identified by its corresponding node, placed at the centre of each segment, at the pile shaft. First $N$ nodes are related to the segments of traction acting on the lateral soilpile surface (barrel loads, see Fig. 2b) while the segment associated to the node $N+1$ refers to the traction acting on the pile-tip (disk load, see Fig. 2c). Therefore, soil-pile interaction will be performed at those points, called coupling points, in where the force equilibrium and compatibility of displacement conditions are complied. The resulting compatibility equations are:

$$
\begin{array}{r}
\mathbf{P}_{p}^{c}+\mathbf{P}_{s}^{c}=0 \\
\mathbf{U}_{p}^{c}=\mathbf{U}_{s}^{c} .
\end{array}
$$

where

$$
\begin{aligned}
\mathbf{P}_{p}^{c} & =\left[\begin{array}{llll}
P_{p}^{c_{1}} & P_{p}^{c_{2}} & \ldots & P_{p}^{c_{N+1}}
\end{array}\right]^{\mathrm{T}}, \\
\mathbf{P}_{s}^{c} & =\left[\begin{array}{llll}
P_{s}^{c_{1}} & P_{s}^{c_{2}} & \ldots & P_{s}^{c_{N+1}}
\end{array}\right]^{\mathrm{T}}, \\
\mathbf{U}_{p}^{c} & =\left[\begin{array}{llll}
U_{p}^{c_{1}} & U_{p}^{c_{2}} & \ldots & U_{p}^{c_{N+1}}
\end{array}\right]^{\mathrm{T}}, \\
\mathbf{U}_{s}^{c} & =\left[\begin{array}{llll}
U_{s}^{c_{1}} & U_{s}^{c_{2}} & \ldots & U_{s}^{c_{N+1}}
\end{array}\right]^{\mathrm{T}} .
\end{aligned}
$$

The subscripts described in equations of the current section are referred to the corresponding substructure of the system (s, soil or p, pile). Coupling points, evaluation point or external force are referred by the superscripts c, e and f, respectively. Thus, in Eq. (1), $\mathbf{P}_{p}^{c}$ and $\mathbf{P}_{s}^{c}$ represent the vectors of vertical interaction forces acting on the pile and the soil, respectively (Eq. (2a),(2b)), while, $\mathbf{U}_{p}^{c}$ and $\mathbf{U}_{s}^{c}$ are referred to the vector of vertical displacements at the coupling points of the pile and soil, respectively (Eq. (2c),(2d)).

\subsection{Response due to an external force applied in the pile head}

Based on the dynamic system presented in the previous section, the displacements at the coupling points when the system is excited by an external vertical force applied in the pile head can be written as

$$
\begin{array}{r}
\mathbf{U}_{p}^{c}=\mathbf{H}_{p}^{c f} F_{p}+\mathbf{H}_{p}^{c} \mathbf{P}_{p}^{c}, \\
\mathbf{U}_{s}^{c}=\mathbf{H}_{s}^{c} \mathbf{P}_{s}^{c},
\end{array}
$$

where the $\mathbf{H}_{p}^{c}$ and $\mathbf{H}_{s}^{c}$ represents the receptance matrices of the pile and the soil, respectively, at the coupling points, having a dimension of $(N+1) \times(N+1)$. The vector $\mathbf{H}_{p}^{c f}$ represent the receptance of the coupling points due to the external force $F_{p}$, which is applied at the pile head. Thus, the interaction forces at the coupling points in the pile can be obtained combining Eq. (3) with Eq. (1), that is

$$
\mathbf{P}_{p}^{c}=-\left(\mathbf{H}_{p}^{c}+\mathbf{H}_{s}^{c}\right)^{-1} \mathbf{H}_{p}^{c f} F_{p} .
$$

Finally, if the dynamic response of the system is required at the pile head, its driving-point response $U_{p}$ is expressed by Eq. (5a), while if the evaluation points is placed on the soil, then its responses $U_{s}$ is defined by Eq. (5b).

$$
\begin{array}{r}
U_{p}=\mathbf{H}_{p}^{e c} \mathbf{P}_{p}^{c}+H_{p}^{e f} F_{p}, \\
U_{s}=-\mathbf{H}_{s}^{e c} \mathbf{P}_{p}^{c},
\end{array}
$$




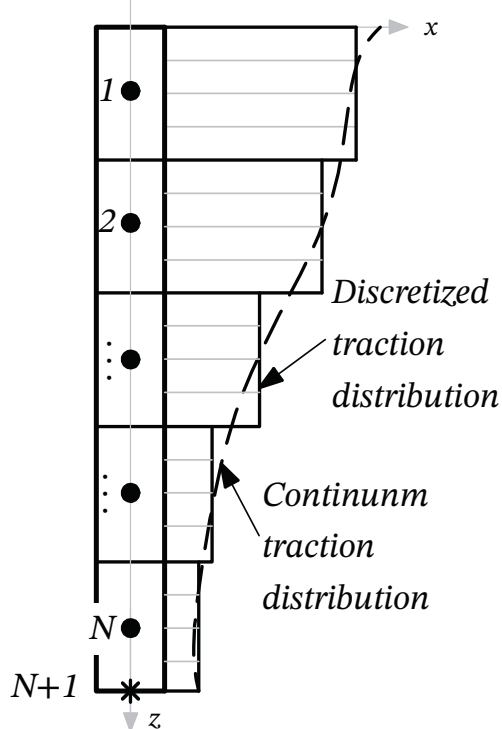

(a)

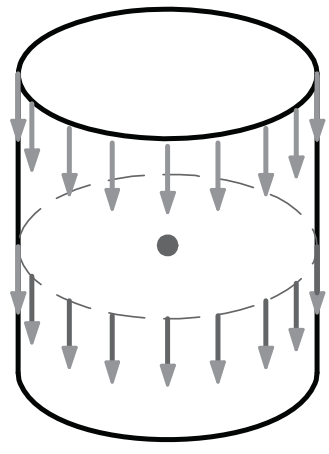

(b)

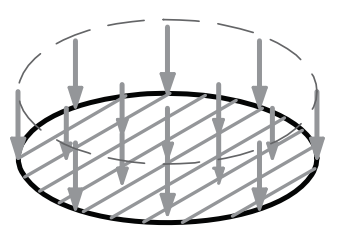

(c)

Figure 2: Discretisation of the pile-soil interaction traction (a), where segment from 1 to $N$ represent to barrel type loads (black dots representing the coupling points) while the last segment $N+1$ depicts the disk type load (coupling point represented by star dot). Continuum barrel traction for the firsts $N$ segments (b). Continuum disk traction for the last segment $N+1$ (c).

where $\mathbf{H}_{p}^{e c}$ and $\mathbf{H}_{s}^{e c}$ refers to the vector of receptances relating the response in the evaluation point, placed at the pile head or at the soil, respectively, due to the interaction forces $\mathbf{P}_{p}^{c}$, while the $H_{p}^{e f}$ is the receptance of the evaluation point due to the external force $F_{p}$ applied in the pile.

\subsection{Response due to an external force applied in the soil}

In the previous section, the system was subjected to an external force placed at the pile head. In the current section, the response of the pile to an incident wave-field due to an external vertical point load in the soil is formulated. In this case, the displacements in the coupling points in the point of view of the pile and the soil are

$$
\begin{array}{r}
\mathbf{U}_{p}^{c}=\mathbf{H}_{p}^{c} \mathbf{P}_{p}^{c}, \\
\mathbf{U}_{s}^{c}=\mathbf{H}_{s}^{c f} F_{s}+\mathbf{H}_{s}^{c} \mathbf{P}_{s}^{c},
\end{array}
$$

respectively, being $F_{s}$ the force applied on the soil and $\mathbf{H}_{s}^{c f}$ the receptance matrix that relates the displacement at the coupling points with the applied external force. Therefore, the corresponding interaction forces of the system are equal to:

$$
\mathbf{P}_{p}^{c}=\left(\mathbf{H}_{p}^{c}+\mathbf{H}_{s}^{c}\right)^{-1} \mathbf{H}_{s}^{c f} F_{s} .
$$

Thus, the dynamic response of the pile at the pile head $U_{p}$ under an incident wave-field produced by $F_{s}$ is given by:

$$
U_{p}=\mathbf{H}_{p}^{e c} \mathbf{P}_{p}^{c}
$$

\subsection{Soil receptance matrices}

In order to compute the soil receptance matrices appearing in the previous sections, the soilpile interaction traction in the point of view of the soil is discretised in a set of $N_{s}$ uniformly 
distributed points placed along the virtual pile perimeter for each pile segment, as shown in Fig. 3. This can be seen as a discretised form of the method proposed by Kaynia and Kausel [3]. The corresponding receptance matrix associated to all these points $\mathbf{H}_{s g}^{c}$ of $M \times M$ dimensions,

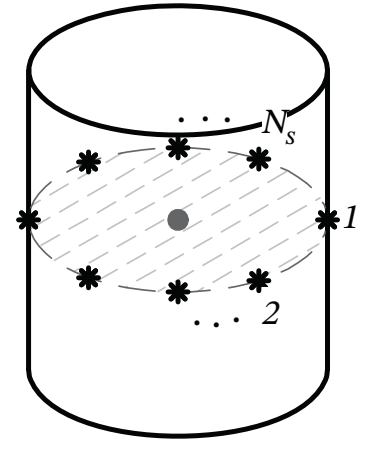

(a)

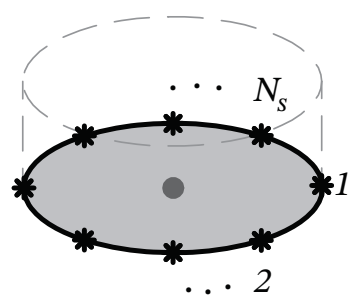

(b)

Figure 3: Traction segment discretised into $N_{s}$ points for a: a) barrel type traction element. b) disk type traction element.

being $M=(N+1) N_{s}$, can be condensed into $\mathbf{H}_{s}^{c}$ by the expression:

$$
\mathbf{H}_{s}^{c}=\mathbf{W}_{d} \mathbf{H}_{s g}^{c} \mathbf{W}_{f}
$$

where $\mathbf{W}_{d}$ is the matrix which condense all $N_{s}$ displacement values of each segment into a corresponding equivalent displacement. The matrix $\mathbf{W}_{f}$ applies the same concept for the case of the interaction forces. In both cases, this research is developed assuming a uniform distribution. Similarly, when an external force is applied in the soil, this procedure can be extended to compute the soil receptance matrix $\mathbf{H}_{s}^{c f}$. Finally, The Green's functions of the soil required to construct $\mathbf{H}_{s g}^{c}$ and the remaining soil receptances are computed using the ElastoDynamics Toolbox for MATLAB [12], which uses the direct stiffness matrix method to deal with wave propagation problems in horizontally layered media.

\subsection{Pile receptance matrices}

The receptance matrices related to the pile can be found assuming that the pile can be modelled as a simple rod structural member. Thus, the differential equation for the axial vibration of the pile in the time domain is described as

$$
m_{p} \frac{\partial^{2} u_{z}}{\partial t^{2}}-E_{p} A_{p} \frac{\partial^{2} u_{z}}{\partial z^{2}}=0
$$

In Eq. (10), the mass per unit of length is defined by $m_{p}$, while $A_{p}$ and $E_{p}$ are the crosssection area and the elastic modulus of the pile, respectively. Therefore, if an external force $F_{z}$ is applied at the pile in some position $z_{1}$ of its shaft, the solution of Eq. (10) in the frequency domain for free-free rod is given by

$$
\begin{array}{cc}
\frac{U_{z}(z)}{F_{z}}=-\frac{\cos (\alpha z)\left[\cos \left(\alpha z_{1}\right)+\sin \left(\alpha z_{1}\right) \tan (\alpha L)\right]}{E_{p} A_{p} \alpha \tan (\alpha L)} & 0 \leq z \leq z_{1}, \\
\frac{U_{z}(z)}{F_{z}}=-\frac{\cos \left(\alpha z_{1}\right) \cos (\alpha z)}{E_{p} A_{p} \alpha \tan (\alpha L)}-\frac{\cos \left(\alpha z_{1}\right) \sin (\alpha z)}{E_{p} A_{p} \alpha} & z_{1} \leq z \leq L,,
\end{array}
$$




$$
\alpha=\frac{m_{p} \omega^{2}}{A_{p} E_{p}},
$$

in where $L$ is the total length of the pile and $\omega$ is the circular frequency in which the dynamic analysis is performed.

\section{COMPARISON OF THE MODEL}

In this section, the accuracy of the new semi-analytical approach is analysed in order to demonstrate its applicability in different pile problems. The methods presented for the comparison are the new semi-analytical approach, the extended Novak approach [10] proposed by Kuo $[7,8]$ and an axisymmetric FEM-based approach with perfect matched layers (aFEM-PML). The aFEM-PML is based on an axissymmetric version of the method presented by [9] considering eight-noded square elements of $25 \mathrm{~cm}$ of side. In the extended Novak approach, the approximate far-field response using the Novak approach is obtained by the assumption that only cylindrical SV-waves can spread from the pile through the soil and just in radial direction $[7,8]$. The parameters used to compute the results are as following: the pile has a length of $L=20 \mathrm{~m}$ and radius $r_{p}=0.354 \mathrm{~m}$ and it is made of concrete with density $\rho_{p}=2800 \mathrm{~kg} / \mathrm{m}^{3}$, Young's modulus $E_{p}=40 \mathrm{GPa}$, Poisson's ratio $\nu_{p}=0.3$ and hysteretic damping ratio $\beta=0.01$; while the soil is modelled as a homogeneous half-space elastic medium with density $\rho_{s}=2250$ $\mathrm{kg} / \mathrm{m}^{3}$, shear modulus $G_{s}=90 \mathrm{MPa}$, Poisson's ratio $\nu_{s}=0.4$ and hysteretic damping ratios for S- and P-waves equals to $D_{s}=0.03, D_{p}=0.03$, respectively. Furthermore, the range of frequency considered to perform the comparison is from $1 \mathrm{~Hz}$ to $80 \mathrm{~Hz}$.

Plots in Fig. 4 show the driving-point response of the pile head. These responses are computed using different values of $N$ and $N_{s}$ and the results are compared with the outcomes from the Novak method. In this figure, a poor agreement in the response is observed when the pile has $N=5$ coupling points, as shown in Fig. 4 a, where the difference between the two methods rise until $11 \mathrm{~dB}$ over the frequency range of $80 \mathrm{~Hz}$. Likewise, if the the number of coupling points is increased to $N=10$ or $N=15$ (see Fig. 4b and Fig. 4c) the response of the system presents a good agreement respect to results obtained by the Novak method (less of $2 \mathrm{~dB}$ of variation) furthermore, the response computed by the current approach shows a smooth curve for the whole range of frequency. Finally, high accuracy is achieved ( $0.6 \mathrm{~dB}$ of variation) when the new semi-analytical method is applied with $N=25$ coupling points, see Fig. 4d. This can be also seen in Fig. 5a, where the results obtained with the aFEM-PML method are also added in the comparison.

In Fig. 5, the new method is compared with the Novak and the aFEM-PML methods in terms of the response at the soil due to the action of a vertical harmonic force at the pile head. The response in the soil has been computed at the ground surface and at distances of $5 \mathrm{~m}, 10 \mathrm{~m}$ and $20 \mathrm{~m}$ from the pile shaft. For this comparison, the new method is applied assuming $N=25$ and $N_{s}=4$. It is observed that the Novak method shows large discrepancies with respect to the aFEM-PML model, mostly at large distances from the pile. That is due to the assumption adopted by Novak in his model and the consequent inaccuracy of the wave-spreading equations [7] in where the wave propagation in the soil due to the axial pile action is assumed to be based only on SV-waves. By other hand, the new semi-analytical model shows less than $2 \mathrm{~dB}, 1.5 \mathrm{~dB}$ and $1 \mathrm{~dB}$ of variations in comparison with the aFEM-PML method when the evaluation point and the pile shaft are at a distance of $5 \mathrm{~m}, 10 \mathrm{~m}$, and $20 \mathrm{~m}$, respectively. This shows that the agreement in the results for axial vibrations is good in the whole frequency range of interest. 

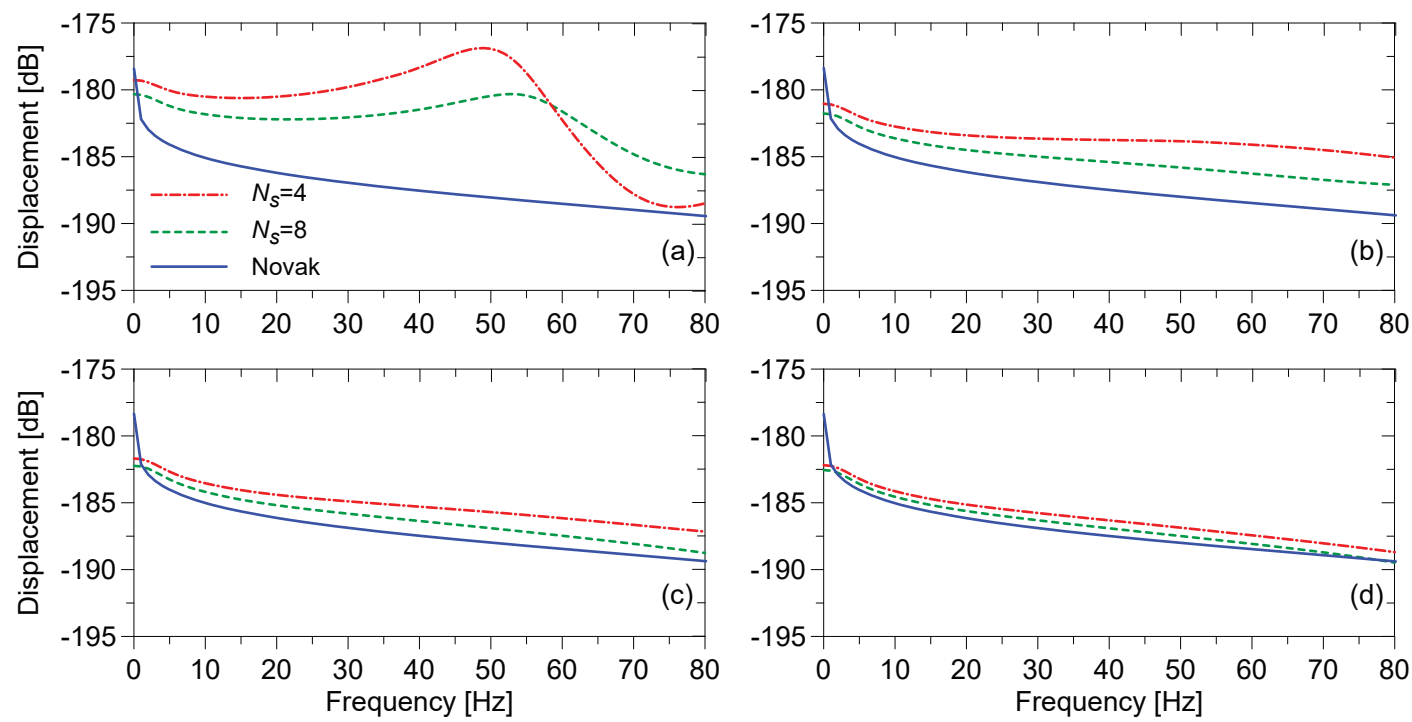

Figure 4: Driving-point response in $\mathrm{dB}$ (with a reference of $1 \mathrm{~m} / \mathrm{N}$ ). Cases: $N=5$ (a), $N=10$ (b), $N=15$ (c) and $N=25(\mathrm{~d})$ coupling points.
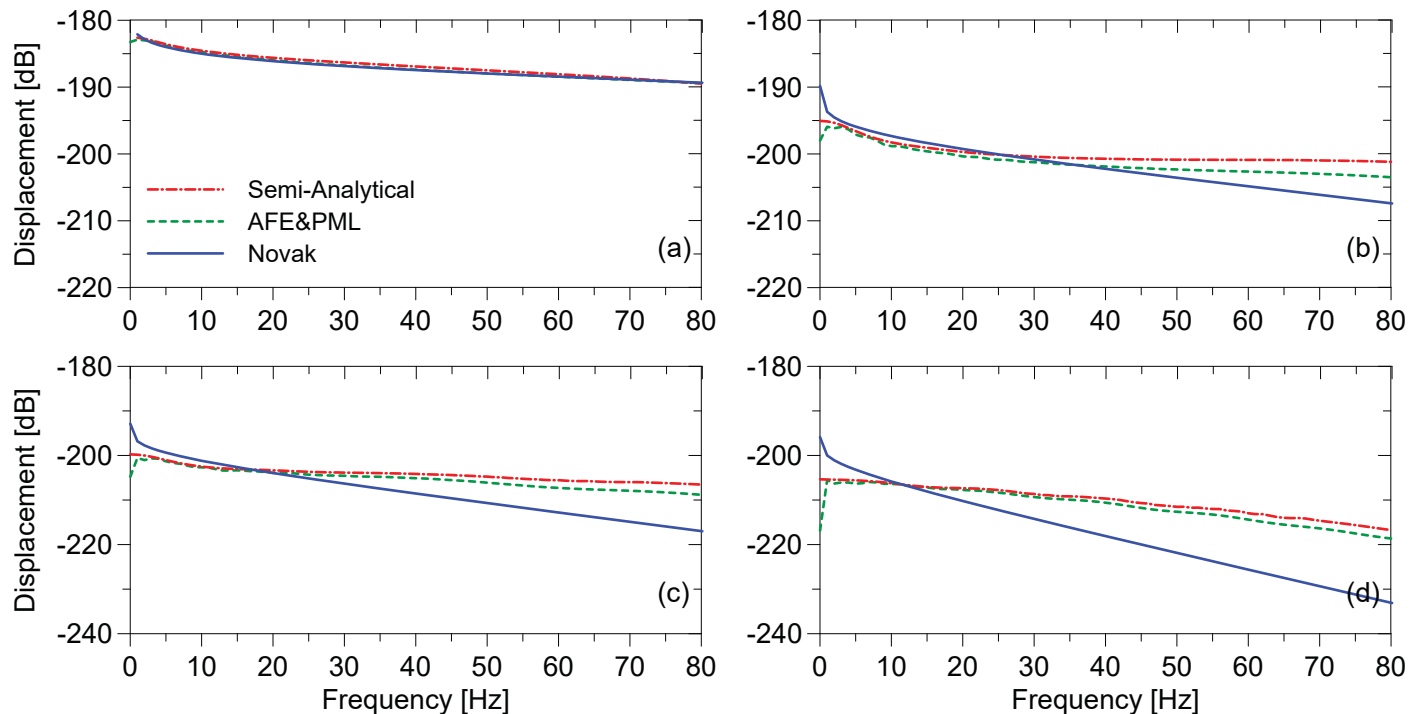

Figure 5: Displacement response in $\mathrm{dB}$ (with a reference of $1 \mathrm{~m} / \mathrm{N}$ ) due to an external force applied at the pile head considering with $N=25$ and $N_{s}=4$ for the semi-analytical approach. Cases: Driving-point response (a); response at $5 \mathrm{~m} \mathrm{(b);} \mathrm{response} \mathrm{at} 10 \mathrm{~m}$ (c); and response at $20 \mathrm{~m}$ (d). 


\section{CONCLUSIONS}

In this paper, a new semi-analytical modelling approach for the dynamic response of a single pile embedded in a half-space is presented. This new semi-analytical model captures the driving-point response of the pile reasonably well, with some differences at high frequencies (less than $1.5 \mathrm{~dB}$ ) when the results obtained are compared against the Novak's method [10]. That agreement is obtained when the number of coupling points is equal to 25 and when the number of points used to discretise the traction distribution of each segment is eight. If the number of coupling points is less than 25 , the differences in the dynamic response are high for the whole range of frequency.

When the response of the soil due to a force in the pile is required, the results obtained with the new approach present a good agreement in comparison with the aFEM-PML model. The aFEM-PML is an accurate numerical model that requires considerable computational costs, while the new approach is able to reach similar levels of accuracy using quite less resources and time, due to its semi-analytical nature. Novak method, in contrast, is showing a poor agreement with respect to the aFEM-PML method, an expected result due to simplifications on soil modelling inherent to the Novak's method. It can be concluded, then, that the new proposed semi-analytical method can provide accurate results on the soil response, being this feature of the method a significant benefit with respect to Novak's method.

\section{ACKNOWLEDGEMENTS}

This research has been carried out with the financial support of three research projects:

- Project VIBWAY: Fast computational tool for railway-induced vibrations and re-radiated noise assessment, with reference RTI2018-096819-B-I00, supported by the Ministerio de Ciencia e Innovación, Retos de Investigación 2018.

- Project NVTRail: Noise and Vibrations induced by railway traffic in tunnels: an integrated approach, funded by FEDER funds through COMPETE2020 (Programa Operacional Competitividade e Internacionalizacão (POCI)) and by national funds (PIDDAC) through FCT/MCTES, with grant reference POCI-01-0145-FEDER-029577.

- Project POCI-01-0145-FEDER-029634 funded by FEDER funds through COMPETE2020 Programa Operacional Competitividade e Internacionalizao (POCI) and by national funds (PIDDAC) through FCT/MCTES.

- The author would like to thank PRONABEC (www • pronabec . gob . pe) for the financial support given through the scholarship: "Beca Presidente de la Repblica" 


\section{REFERENCES}

[1] A. J. Deeks and M. F. Randolph. Axisymmetric time-domain transmittings boundaries. Journal of Engineering Mechanics, 120(1):25-42, 1994.

[2] J. A. Forrest and H. E. M. Hunt. A three-dimensional tunnel model for calculation of train-induced ground vibration. Journal of Sound and Vibration, 294(4-5):678-705, jul 2006.

[3] A. M. Kaynia and E. Kausel. Dynamics of piles and pile groups in layered soil media. Soil Dynamics and Earthquake Engineering, 10(8):386-401, 1991.

[4] R. Kuhlemeyer. Static and dynamic laterally loaded floating piles. Journal of the Geotechnical Engineering Division, Proceedings of the American Society of Civil Engineers, 105(GT2):289-304, 1979.

[5] R. Kuhlemeyer. Vertical vibration of piles. Journal of the Geotechnical Engineering Division, Proceedings of the American Society of Civil Engineers, 105(GT2):273-287, 1979.

[6] K. Kuo and H. Hunt. Dynamic models of piled foundations. Applied Mechanics Reviews, 65(May 2013):9, 2013.

[7] K. A. Kuo. Vibration from underground railways: considering piled foundations and twin tunnels. PhD thesis, University of Cambridge, 2010.

[8] K. A. Kuo and H. E. M. Hunt. An efficient model for the dynamic behaviour of a single pile in viscoelastic soil. Journal of Sound and Vibration, 332(10):2549-2561, may 2013.

[9] P. Lopes, P. A. Costa, M. Ferraz, R. Calçada, and A. S. Cardoso. Numerical modeling of vibrations induced by railway traffic in tunnels: From the source to the nearby buildings. Soil Dynamics and Earthquake Engineering, 61-62:269-285, jun 2014.

[10] M. Novak. Dynamic stiffness and damping of piles. Canadian Geotechnical Journal, 11(4):574-598, 1974.

[11] E. Ntotsios, W. I. Hamad, D. J. Thompson, M. F. M. Hussein, H. E. M. Hunt, and J. P. Talbot. Predictions of the dynamic response of piled foundations in a multi-layered half-space due to inertial and railway induced loadings. In 5th ECCOMAS Thematic Conference on Computational Methods in Structural Dynamics and Earthquake Engineering, 2015.

[12] M. Schevenels, S. François, and G. Degrande. EDT: An ElastoDynamics Toolbox for MATLAB. Computers and Geosciences, 35(8):1752-1754, 2009. 